\title{
A novel inhibitor of Plasmodium falciparum spermidine synthase: a twist in the tail
}

Pieter B Burger ${ }^{1}$, Marni Williams', Janina Sprenger ${ }^{2}$, Shaun B Reeksting ${ }^{1}$, Mariëtte Botha ${ }^{1}$, Ingrid B Müller ${ }^{3,4}$, Fourie Joubert ${ }^{1}$, Lyn-Marie Birkholtz ${ }^{1}$ and Abraham I Louw ${ }^{1 *}$

\begin{abstract}
Background: Plasmodium falciparum is the most pathogenic of the human malaria parasite species and a major cause of death in Africa. It's resistance to most of the current drugs accentuates the pressing need for new chemotherapies. Polyamine metabolism of the parasite is distinct from the human pathway making it an attractive target for chemotherapeutic development. Plasmodium falciparum spermidine synthase (PFSpdS) catalyzes the synthesis of spermidine and spermine. It is a major polyamine flux-determining enzyme and spermidine is a prerequisite for the post-translational activation of $P$. falciparum eukaryotic translation initiation factor 5A (elF5A). The most potent inhibitors of eukaryotic SpdS's are not specific for PfSpdS.

Methods: 'Dynamic' receptor-based pharmacophore models were generated from published crystal structures of SpdS with different ligands. This approach takes into account the inherent flexibility of the active site, which reduces the entropic penalties associated with ligand binding. Four dynamic pharmacophore models were developed and two inhibitors, (1R,4R)-(N1-(3-aminopropyl)-trans-cyclohexane-1,4-diamine (compound 8) and an analogue, $\mathrm{N}$-(3-aminopropyl)-cyclohexylamine (compound 9), were identified.
\end{abstract}

Results: A crystal structure containing compound 8 was solved and confirmed the in silico prediction that its aminopropyl chain traverses the catalytic centre in the presence of the byproduct of catalysis, $5^{\prime}$-methylthioadenosine. The $I_{50}$ value of compound 9 is in the same range as that of the most potent inhibitors of PfSpdS, S-adenosyl-1,8-diamino-3-thio-octane (AdoDATO) and 4MCHA and 100-fold lower than that of compound 8. Compound 9 was originally identified as a mammalian spermine synthase inhibitor and does not inhibit mammalian SpdS. This implied that these two compounds bind in an orientation where their aminopropyl chains face the putrescine binding site in the presence of the substrate, decarboxylated S-adenosylmethionine. The higher binding affinity and lower receptor strain energy of compound 9 compared to compound 8 in the reversed orientation explained their different $I C_{50}$ values.

Conclusion: The specific inhibition of PfSpdS by compound 9 is enabled by its binding in the additional cavity normally occupied by spermidine when spermine is synthesized. This is the first time that a spermine synthase inhibitor is shown to inhibit PfSpdS, which provides new avenues to explore for the development of novel inhibitors of PfSpdS.

Keywords: Spermidine synthase, Plasmodium falciparum, Virtual screening, Spermidine, Spermine, Pharmacophores, Crystal structures

\footnotetext{
* Correspondence: braam.louw@up.ac.za

'Department of Biochemistry, UP Centre for Sustainable Malaria Control, Faculty of Natural and Agricultural Sciences, University of Pretoria, Private Bag X20, Hatfield 0028, South Africa

Full list of author information is available at the end of the article
} 


\section{Background}

Plasmodium falciparum is the most pathogenic of the human malaria species with approximately 207 million cases in 2012 and an estimated 627,000 deaths. The majority of the mortalities occur in Africa, mostly in children under the age of five and pregnant women. Anti-malarial drug resistance is a major concern especially against the artemisinins (the last remaining fully-effective antimalarial) where resistance has recently been detected in Southeast Asia [1]. No new classes of anti-malarials have been introduced into clinical practice since 1996 and there is no vaccine available. A pressing need therefore exists to identify novel targets for new anti-malarial development [2].

The inhibition of polyamine biosynthesis has been widely studied as a target for antiproliferative therapy with some success in cancer prevention and treatment, but most notably in the treatment of West African sleeping sickness [3]. Polyamines are ubiquitous aliphatic amines that are essential for cell growth, proliferation and differentiation in the majority of living cells $[4,5]$. The major polyamines putrescine, spermidine and spermine are synthesized by ornithine decarboxylase (ODC, EC 4.1.1.17), spermidine synthase (SpdS; EC 2.5.1.16) and spermine synthase (SpmS, EC 2.5.1.22), respectively. The synthesis of spermidine and spermine requires decarboxylated $S$-adenosylmethionine (dcAdoMet) as aminopropyl donor, which is produced by $S$-adenosylmethionine decarboxylase (AdoMetDC, EC 4.1.1.50). The P. falciparum polyamine biosynthesis pathway has several unique and exploitable parasite-specific characteristics such as the association of the pathway-regulating enzymes, AdoMetDC and ODC, in a heterotetrameric bifunctional protein [6,7] and the absence of a polyamine interconversion pathway $[7,8]$.

Accumulating evidence has highlighted the potential of several enzymatic activities involved in the P. falciparum polyamine pathway as targets for the development of anti-malarial chemotherapeutics $[9,10]$. The ensemble of polyamines increases during the asexual, intra-erythrocytic developmental cycle and occurs in millimolar concentrations within the parasite [11-13]. Spermidine levels of the intra-erythrocytic parasite exceed that of the other polyamines, emphasizing the role of PfSpdS as a major polyamine flux-determining enzyme [11]. In addition, spermidine appears to have greater metabolic importance since it is a prerequisite for the post-translational activation of $P$. falciparum eukaryotic translation initiation factor 5A (elF5A), which is required for protein synthesis [9,14-17]. The biosynthesis of low concentrations of spermine has been attributed to a minor, secondary activity of PfSpdS since there is no evidence for a $P$. falciparum equivalent to SpmS [18].
The crystal structures of several SpdS have been solved and released in the PDB, which include human, Escherichia coli, and plant SpdS [19]. The PfSpdS structure was first described by Dufe et al. [20] and consists of two domains including an $\mathrm{N}$-terminal $\beta$-strand (six antiparallel strands) and a central catalytic domain with a sevenstranded $\beta$-sheet flanked by nine $\alpha$-helices forming a Rossmann-like fold, which is typical of methyltransferases and nucleotide-binding proteins. The active site is located between the $\mathrm{N}$ - and $\mathrm{C}$-terminal domains and is divided into distinct binding cavities for its substrates dcAdoMet and putrescine, which is common for all SpdS. The active site is spanned by a so-called gatekeeper loop that is only structured when ligands are bound.

Several SpdS inhibitor studies have been performed in the last decades, with the most potent inhibitors of eukaryotic SpdS's being two multi-substrate or transition state analogues, $S$-adenosyl-1,8-diamino-3-thio-octane (AdoDATO) and [3-(R,S)-(5'-deoxy-5'-carbaadenos-6' yl)-spermidine] (adenosylspermidine) [21], which bind to both substrate binding cavities. A potent inhibitor of PfSpdS, trans-4-methylcyclohexylamine (4MCHA), was derived from a structure-activity relationship (SAR) study of the putrescine binding cavity, which highlighted the hydrophobic and hydrogen bond-donating pharmacophore features corresponding to the primary alkyl component and non-attacking nitrogen of putrescine, respectively [22]. The X-ray structure of the complex [PDB:2PT9] demonstrated that 4MCHA, only binds in the putrescine binding cavity when dcAdoMet is present [20]. In addition, 12 other crystal structures of PfSpdS have been resolved including one co-crystallized with AdoDATO [PDB:2I7C] [20].

In the first structure-based drug design study of PfSpdS, the information obtained from the crystal structure with AdoDATO was used to generate pharmacophore models [23]. Virtual screening of an in-house chemical library resulted in the identification of $28 \mathrm{com}$ pounds as active site binders but no significant inhibitors. In the present structure-based study a 'dynamic' receptor-based pharmacophore model was developed to identify potential inhibitors of PfSpdS. This approach takes into account the inherent flexibility of the active site, which reduces the entropic penalties associated with ligand binding $[24,25]$. Subsequent co-crystallization of PfSpdS with MTA and two potential inhibitors yielded one crystal structure with compound 8 that validated the in silico predicted interactions, i.e., the aminopropyl tails of these compounds cross the catalytic centre and bind into the aminopropyl cavity of the dcAdoMet site. However, the 100-fold better inhibition by compound 9 compared to compound 8 could only be explained by their binding in a reversed orientation in the presence of 
dcAdoMet with their aminopropyl tails facing the nonattacking side of the putrescine/spermidine binding cavity. Compound 9 is thus predicted to inhibit PfSpdS by capitalizing on its ability to produce spermine, which concur with previous literature reports that compound 9 is an inhibitor of mammalian spermine synthase but not mammalian SpdS [22]. Therefore compound 9 acts as a spermidine analogue and specifically inhibits PfSpdS due to its unique ability to also accept spermidine as substrate for spermine synthesis.

\section{Methods}

\section{Protein structure quality assessment}

The structural integrity and quality of the [PDB:2I7C] and [PDB:2PT9] PfSpdS crystal structures were analysed with WHATIF [26] and PROCHECK [27]. pKa predictions were performed using UHBD [28] and YASARA [29] on the [PDB:2HTE] and [PDB:2I7C] PfSpdS crystal structures and included the solvent molecules. The pKa predictions were performed by adjusting the default scripts in the UHBD package as follows: the pKa values were predicted at a $\mathrm{pH}$ of 7.0, the temperature was set to $293 \mathrm{~K}$ and the dielectric constants of the solvent and protein were kept at 80 and 20, respectively. Lastly, the protonation states of the His residues were assigned based on consensus decisions (His103HSD, His108HSE, His236HSE and His304HSE).

\section{Phase space sampling}

The molecular dynamics (MD) simulations and energy minimization were performed with NAMD [30] using the CHARMM force field [31] in the absence of AdoDATO. The dimension of the water box was set to $79 \times 100 \times 81 \AA$ with the water boundary set at a distance of $10 \AA$ from the protein. In preparation for the PfSpdS equilibration run the protein was protonated according to the pKa values obtained above. Steepest descent (SD) minimization consisting of 100 steps was performed on the added hydrogens followed by solvation and neutralization of the protein using VMD [32]. The water and ions in the water box were subsequently minimized and equilibrated. This was done for 2,000 steps of SD minimization followed by a 20 picosecond (ps) equilibration of the water and ions with a reassignment of velocities every 1 ps. This step was followed by minimization of the protein for 200 steps using SD while keeping the solvent fixed. The protein and solvent were then heated to $310 \mathrm{~K}$ with a heating gradient of $10 \mathrm{~K}$ every 500 steps $(1 \mathrm{ps})$ after which the protein was kept at $310 \mathrm{~K}$ for $34 \mathrm{ps}$ resulting in a total duration of the heating process of $50 \mathrm{ps}$. The protein was then equilibrated for 500 ps and the temperature reassigned every 500 steps to $310 \mathrm{~K}$. Periodic boundary conditions (PBC) were applied and all the electrostatic interactions were included using the Particle Mesh Ewald (PME) summation method [33]. Constant pressure and temperature control was applied using the Berendsen method [34]. The production run was performed under PBC conditions. As with the equilibration run, Berendsen dynamics were used to perform constant pressure and temperature control.

The resulting trajectory was clustered with the gromos algorithm [35] of the g_cluster module from GROMACS [36] to obtain the central structure of the representative clusters. The clustering was performed separately for each monomer of the simulated dimer. The MD trajectory was aligned and clustered based on the active site of each of the monomers. The cut-off values used for the clustering were $1.15 \AA$ and $1.14 \AA$ for chain $\mathrm{B}$ and $\mathrm{C}$, respectively. The isomiddle structures of the top five representative clusters of both monomers were selected and compared relative to root mean square deviation (RMSD). From these structures five were selected to best represent the RMSD range between the structures and subsequently used in further studies.

\section{Negative image construction and hit analysis}

The sub-ensemble of structures selected in the previous section was prepared for molecular interaction field (MIF) analysis, which was performed with GRID [37]. A grid box was generated to cover the active site with the following dimensions: TOPX 19.82, BOTX 16.73; TOPY 16.64, BOTY 20.85; TOPZ 19.28 and BOTZ 14.02. Two water-binding hotspots were identified and subsequently added to the sub-ensemble of structures. MIF analysis was performed to explore the chemical nature of the active site by using probes representing hydrogen bond donors (HBD), hydrogen bond acceptors (HBA) and hydrophobic (HYD) PhFs. Three different HBD probes were used and included the N2 (a neutral flat nitrogen with two hydrogens $(\mathrm{NH} 2)$ ), $\mathrm{N} 1+$ (a sp amine $(\mathrm{NH})$ cation) and $\mathrm{N} 3+$ (a sp amine (NH) cation) probes. For the identification of HBA binding hotspots three probes were used and included the $\mathrm{O}$ (a sp carbonyl oxygen), $\mathrm{O} 1$ (an alkyl hydroxy $(\mathrm{OH})$ group) and $\mathrm{N}:=$ (a sp2 nitrogen with lone pair of electrons) probes. The HYD features were identified using both the DRY (a general hydrophobic probe) and Me (the $\mathrm{C} 3$ probe represents a $\mathrm{CH}_{3}$ chemical moiety) probes. The default GRID parameters were used except that the number of planes of grid points per Angstrom (NPLA) and ALMD parameters were changed to four (spacing the grid points $0.25 \AA$ apart) and one (print additional data in the output files), respectively.

\section{Pharmacophore model selection, screening and docking}

The binding energy values and coordinates for all the respective probes were extracted from the GRID calculations for each of the structures from the sub-ensemble 
and were represented in Cartesian space. Grid point clusters were identified and selected by visualization of the grid points within the active site. The PhFs were generated by extracting the attributes from the grid point clusters and calculating their centre of mass (energy-weighted) as well as their radius of gyration. This was performed on all the probes used in MIF analysis, using an in-house python script that generates a PDB file containing the centre of mass (representing the $\mathrm{PhF}$ coordinates; energy-weighted), the radius of gyration and the mean energy of the PhF. The PhFs identified for the sub-ensemble and those from the [PDB:2PT9] crystal structure were treated separately. Atoms to represent the exclusion volumes (EVs) for the DPM were selected after visual inspection of the residues surrounding the active site. The coordinates for these atoms were extracted from all the structures in the subensemble. The centre of mass for these atoms was calculated to include the dynamic behaviour of the active site and thus represent 'dynamic' EVs, which was also performed using an in-house python script.

For the selection of DPMs the active site was subdivided into four regions, DPM1 to DPM4. For each of these regions various DPMs represented by different combinations of PhFs were constructed. The DPMs were built in Catalyst v4.10 (Accelrys). The EVs were added using Discovery Studio 2.0 before screening subsets of the ZINC database. The drug-like subset of the ZINC database was used to construct a multi-conformer composite database and was generated using catDB (Accelrys). During database construction the maximum number of conformers was limited to 250. Catalyst v4.10 was used to search both the constructed databases selecting the best flexible search parameter. The DPMs used during searching included the EVs generated from the sub-ensemble of PfSpdS. Compounds identified during these searches were fitted to its DPM to get the best fitting molecules and these were ranked accordingly. Visual inspection of the best-fitting compounds was performed to select the top compounds based on their fit values and orientation within the active site. Selected compounds were initially docked using AutoDock v4.0. Two different PfSpdS monomers were prepared for docking and included chain $\mathrm{C}$ of [PDB:2I7C] and chain A of [PDB:2PT9], following 400 steps of steepest decent energy minimization. The AutoDockTools (ADT) kit was used in preparation of both the target structures and identified compounds. The following parameters were changed for docking: the Genetic Search Algorithm was selected and the number of genetic algorithm runs changed to 50; the Translation parameter was changed to $0.2(\AA /$ step); both the Quaternion and Torsion parameters were changed to 5 (degree/step); and, the RMS Cluster tolerance to $1.5 \AA$. The docked compounds were evaluated based on their energy scores and poses within the active site of both the [PDB:2PT9] and [PDB:2I7C] PfSpdS crystal structures used during docking.

The binding poses of the selected compounds were refined using Glide (Schrödinger, Inc) as docking engine. The compounds were prepared for docking by predicting their protonation states at $\mathrm{pH} 7.4$ by using the program Epik (Schrödinger, Inc.). The docking grids were generated for PfSpdS ([PDB:2I7C]; chain B) using Asp196 as the centre with the grid size set to $33 \AA$, and the ligand diameter midpoint box was set to $14 \AA$ for all three axes. The extra precision scoring algorithm from Glide was used to identify the best scoring compounds during docking. All ligands were treated as flexible during docking, allowing for sampling of nitrogen inversion and ring conformations. Docking poses were restricted to ten poses per ligand followed by postdocking minimization (minimization was performed with the Optimized Potentials for Liquid Simulations-all atoms force field) with a rejection threshold of $0.5 \mathrm{kcal} / \mathrm{mol}$. The structures of the best-scoring docked pose for each of the compounds are denoted in Figure 1 and in Additional file 1. MM/GBSA calculations were performed using Prime to determine the binding free energies of selected ligands (Schrödinger, Inc). Protein flexibility was incorporated for residues within $12 \AA$ of the selected ligands.

\section{Enzyme inhibition assays}

Compounds tested for inhibition of PfSpdS (Figure 1, Table 1 and Additional file 1) were supplied by the following manufacturers: compound 1 by Matrix Scientific (Columbia, South Carolina, USA), compound 2 (purity 97\%) by CHEM IMPEX International, Inc (Wooddale, Illinois, USA), compounds 3, 4, 7 and 9 (purity 98\%) by TCI Europe (Tokyo, Japan), compound 8 (purity 97.7\%) was synthesized by PharmaAdvance Inc (China) and 4MCHA was a kind gift from Keijiro Samejima (Josia University, Saitama, Japan). A 29 amino acid N-terminus deletion mutant of PfSpdS cloned into pTRCHisB (Invitrogen) was expressed in E. coli BLR (DE3), purified and assayed as described by Haider et al. [18]. In later studies, an improved developing medium for TLC separations consisting of iso-propanol, acetic acid and water saturated with $\mathrm{NaCl}(62.5: 15: 17.5, \mathrm{v} / \mathrm{v} / \mathrm{v})$, was employed. Initial inhibition assays were performed with $100 \mu \mathrm{M}$ of each of the compounds before their selection for $\mathrm{IC}_{50}$ determinations. Stock solutions of the inhibitors were prepared in $\mathrm{ddd}_{2} \mathrm{O}$. For the $100 \mu \mathrm{M}$ assays, $1 \mu \mathrm{g}$ purified PfSpdS, $50 \mu \mathrm{M}$ RS-dcAdoMet, $100 \mathrm{nC}_{\mathrm{i}}\left[2,3-{ }^{3} \mathrm{H}\right]$ putrescine hydrochloride $\left(60 \mathrm{C}_{\mathrm{i}} / \mathrm{mmol}\right.$; American Radiolabelled Chemicals, Inc, Minnesota, USA) and $50 \mu \mathrm{M}$ unlabelled putrescine dihydrochloride (Sigma) were used. For $\mathrm{IC}_{50}$ determinations $0.5 \mu \mathrm{g}$ purified PfSpdS, $350 \mu \mathrm{M}$ RS-dcAdoMet $\left(5 \mathrm{xK}_{\mathrm{m}}\right)$ and $250 \mu \mathrm{M}$ unlabeled putrescine hydrochloride $\left(5 \mathrm{xK}_{\mathrm{m}}\right)$ and a range of concentrations of 


\begin{tabular}{|c|c|c|c|c|}
\hline Compound & Structure & $\begin{array}{l}\text { Best Fit } \\
\text { Value/number } \\
\text { of PhFs }\end{array}$ & $\begin{array}{l}\text { Docking } \\
\text { scores } \\
(\mathrm{kcal} / \mathrm{mol})\end{array}$ & $\begin{array}{l}\text { \% } \\
\text { Inhibition } \\
(\text { at } 100 \\
\mu \mathrm{M})^{\mathrm{a}}\end{array}$ \\
\hline $4 \mathrm{MCHA}^{\mathrm{b}}$ & & $0.98 / 2 *$ & -6.2 & $95.3 \pm 0.4$ \\
\hline $1^{\mathrm{b}}$ & & $0.53 / 3$ & -7.4 & $17 \pm 9.4$ \\
\hline $2^{\mathrm{b}}$ & & NA & -8.3 & $6.9 \pm 5.8$ \\
\hline AdoDATO & & $0.66 / 4 *$ & -17.1 & ND \\
\hline Adenosylspermidine $\mathrm{e}^{\#}$ & & $0.55 / 5^{*}$ & -22.3 & ND \\
\hline
\end{tabular}

compound 9 (7.5-120 $\mu \mathrm{M})$, compound 8 (125-1,000 $\mu \mathrm{M})$, and 4MCHA $(0.5-64 \mu \mathrm{M})$ were used for $\mathrm{IC}_{50}$ determinations. All inhibition assays were performed in triplicate.

\section{Protein purification and crystallization of PfSpdS}

For protein crystallization of PfSpdS the gene corresponding to a protein lacking 39 amino acids at the $\mathrm{N}$-terminus, and cloned into the p15-TEV-LIC vector, was obtained from SGC Toronto [40]. As described by Dufe et al., this truncation is crucial to obtain protein crystals that can be used for structure determination [20]. The protein expression and isolation was followed according to Dufe et al. [20]. His-tag cleavage of the purified protein with Pro-TEV protease (Promega) was performed overnight at $4^{\circ} \mathrm{C}$ in the presence of $1 \mathrm{mM}$ DTT. The cleaved protein was purified with Ni-NTA (Sigma-Aldrich) affinity chromatography and buffer exchange was performed in crystal buffer $(10 \mathrm{mM}$ Hepes, $\mathrm{pH} 7.5,500 \mathrm{mM}$ $\mathrm{NaCl}$ ). The protein was concentrated to $22.8 \mathrm{mg} / \mathrm{mL}$ and stored at $4^{\circ} \mathrm{C}$.

PfSpdS was crystallized in the presence of compound 8 and compound 9 as well as with and without MTA using the hanging drop vapour diffusion method at $293 \mathrm{~K}$. Protein solution at a concentration of $5 \mathrm{mg} / \mathrm{ml}$ was mixed with reservoir solution containing 25\% PEG3350, $0.1 \mathrm{M}$ MES pH 5.6 and $0.1 \mathrm{M}\left(\mathrm{NH}_{4}\right)_{2} \mathrm{SO}_{4}$. The PfSpdS inhibitorMTA crystals were obtained by pre-incubation of $5 \mathrm{mg} /$ $\mathrm{mL}$ protein $(1 \mu \mathrm{L})$ with $2.5 \mathrm{mM}$ of compound and MTA each for $30 \mathrm{~min}$ at $298 \mathrm{~K}$ followed by mixing with $1 \mu \mathrm{L}$ of reservoir solution. Crystals were transferred to cryoprotectant solution containing the reservoir solution and $15 \%$ glycerol prior to freezing directly in a nitrogen 
Table 1 Effects of various inhibitors on Plasmodium falciparum spermidine synthase

\begin{tabular}{lll}
\hline Inhibitor & $\begin{array}{l}\text { Mammalian } \\
\text { Enzyme inhibition, } \\
\mathbf{I C}_{\mathbf{5 0}}(\boldsymbol{\mu M})\end{array}$ & $\begin{array}{l}\text { P. falciparum } \\
\text { Enzyme inhibition, } \\
\mathbf{I C}_{\mathbf{5 0}}(\boldsymbol{\mu M})\end{array}$ \\
\hline 4MCHA & $1.7^{\dagger}$ & $1.4 \pm 0.1^{*}$ \\
Cyclohexylamine & $8.1^{\S}$ & $19.7 \pm 3.1^{*}$ \\
APE & $1.7^{\perp}$ & $6.5 \pm 2.1^{*}$ \\
AdoDATO & $0.05-0.10^{\dagger}$ & $8.5^{\#}$ \\
Adenosylspermidine & $0.014^{\dagger}$ & N.D. \\
MTA & N.D & $159 \pm 27^{*}$ \\
Compound 8 & N.D. & $619 \pm 144^{\mathrm{a}}$ \\
Compound 9 & N.D. & $7.4 \pm 2.4^{\mathrm{a}}$ \\
\hline
\end{tabular}

${ }^{a}$ Results are the mean of at least three independent,

duplicate experiments \pm SEM.

*Haider et al. [18].

\#Dufe et al. [20].

${ }^{\$}$ Shirahata et al. [22].

${ }^{\dagger}$ Lakanen et al. [21]; Pegg et al. [38]

${ }^{\perp}$ Goda et al. [39].

cryostream at $100 \mathrm{~K}$. Data was collected at beamline I911-2 (MAX Lab, Lund, Sweden) and processed using the XDS package [41].

Molecular replacement was performed with CNS (v1.2) [42] using the PfSpdS-MTA structure [PDB:2HTE] as search model with subsequent refinement using REFMAC [43] with model building in COOT [44]. The library files for compound 8 were generated using the PRODRG server [45]. The main chain polypeptide conformations of the crystal structures were verified by Ramachandran plots using RAMPAGE [46]. Model quality was checked with PROCHECK and the Joint Structural Genomics Consortium Quality Control v2.7, which contains MolProbity [47] and ADIT [48] checks.

\section{Results}

Protein phase space sampling and clustering

Phase space sampling was used to explore the protein conformational landscape in order to identify a diverse set of conformations over time representative of the active site in solution. An ensemble of protein binding sites has been shown to increase enrichment and identification of a diverse set of ligands when used in virtual screens over a single protein conformation [49]. In this study a MD simulation was used as the phase space sampling method to estimate the equilibrium and dynamic properties of the PfSpdS dimer co-crystallized with AdoDATO [PDB:2I7C]. A 5 nanosecond (ns) MD simulation of the ligand bound enzyme showed similar RMSDs for subunits $B$ and $C$ in the first $2.5 \mathrm{~ns}$ (Additional file 2). There was a slight increase in the backbone RMSD of subunit $\mathrm{C}$ over the last $2.5 \mathrm{~ns}$ compared to subunit $B$ due to increased movement of the gate-keeping loop. Subunits B and C were therefore treated as two separate trajectories and clustered accordingly. Clustering of the trajectory was based on 62 residues identified within $7 \AA$ of AdoDATO in the binding cavity and used to obtain at least $90 \%$ of the MD trajectory frames into the five most populated clusters (93.4 and 99.1\% for subunits B and C, respectively). The iso-middle structures of the top five clusters for both monomers were selected and their structural diversities were compared based on the RMSD values of their active sites (Table 2). Six of the ten structures were selected based on these RMSD values as representative of the sampled conformations (96.2\% of the total sampled space) forming a sub-ensemble of structures (Table 2) and subsequently used for negative image construction.

Conformational changes within the putrescine-binding cavity were determined by comparing the starting structure [PDB:2I7C] and the sub-ensemble of structures. The most prominent feature noted is the conformational change of Gln229 in the absence of AdoDATO; the sidechain amide group orientates perpendicular in the ligand free state compared to the orientation with the ligand bound state, which corresponds to the crystal structure of the ligand free state [PDB:2PSS] (Additional file 3). A superimposition of the ligand free PfSpdS sub-ensemble on the putrescine-bound human SpdS [PDB: 2O06] structure and the PfSpdS crystal structures bound with AdoDATO as well as 4MCHA-dcAdoMet showed that Gln229 assumes a nearly identical orientation for all of these structures (Additional file 3). Importantly, this

Table 2 Clustering of the sampled phase space

\begin{tabular}{llll}
\hline Protein subunit & $\begin{array}{l}\text { C }_{\mathbf{\alpha}} \text {-backbone } \\
\text { RMSD (Å) }\end{array}$ & $\begin{array}{l}\text { Active site } \\
\text { RMSD (Å) }\end{array}$ & \% representation \\
\hline Cluster 1B ${ }^{\#, *}$ & 0.00 & 0.00 & 67.74 \\
Cluster 2B* & 1.46 & 1.86 & 19.20 \\
Cluster 3B* & 1.19 & 1.32 & 6.62 \\
Cluster 4B & 1.45 & 1.89 & 0.24 \\
Cluster 5B* & 0.72 & 0.69 & 0.22 \\
Cluster 1C* & 1.67 & 2.25 & 34.24 \\
Cluster 2C & 1.56 & 1.99 & 26.32 \\
Cluster 3C & 1.59 & 2.00 & 19.32 \\
Cluster 4C & 1.38 & 1.74 & 6.69 \\
Cluster 5C* & 1.80 & 2.40 & 6.74 \\
PDB:2PT9A & 1.56 & 1.99 & - \\
PDB:2PT9B & 1.65 & 2.17 & - \\
PDB:2PT9C & 3.77 & 3.68 & - \\
\hline Reference stucture. & & & \\
\hline
\end{tabular}

\#Reference structure.

*Structures selected to represent the sub-ensemble.

The protein subunits represent the top five clusters of subunits $B$ and $C$ from $M D$. The representation of the sampled space of each cluster from the MD is given as a percentile of the total sampled space per subunit. The subunits of the ligand bound crystal structure [PDB:2PT9] with its $C_{a}$-backbone and active site RMSD values are included. 
conformational change significantly alters the binding characteristics of the putrescine-binding cavity and introduces a series of caveats in the identification of pharmacophore features ( $\mathrm{PhFs}$ ) during negative image construction. Therefore, in addition to the sub-ensemble of structures, the three protein chains of PfSpdS cocrystallized with 4MCHA and dcAdoMet [PDB:2PT9] were included in the negative image construction to identify PhFs representing both the ligand bound and ligand free states (Table 2). The 5 ns MD simulation is only able to sample a small part of the conformational energy landscape, however, in combination with the ligand-bound crystal structures it covers a diverse set of structural conformations [49].

\section{Negative image construction}

The process of generating $\mathrm{PhFs}$ representative of small molecules using a protein structure is known as negative image construction. Probes descriptive of HBD, HBA or HYD characteristics are placed within the binding cavity and used to identify the most favourable binding hotspots for the PhFs. These PhFs are then selected to generate pharmacophore models that can be used in virtual screening of small molecule chemical libraries. Using this methodology a large number of PhFs were identified, which allowed the selection of a set of pharmacophore models to be used in virtual screening.

Energetically favourable binding hotspots were identified by molecular interaction field (MIF) analysis and used to derive the initial PhFs. These PhFs were compared to the known binding features obtained from the PfSpdS structures co-crystallized with inhibitors. Eight different probes representing HBA, HBD and HYD characteristics were used in MIF analysis (see Methods). The resulting grid points representative of the favourable binding areas were extracted and overlaid. Clusters from these grid points were identified and selected to represent $\mathrm{PhFs}$ (binding hotspots) if they represented at least $60 \%$ of the sub-ensemble of structures. The binding energies associated with each of the grid points were assigned as a weight and the centre of masses were calculated to determine an energy-weighted centre of the cluster (ranging between -1.3 for the HYD probes to $-24.0 \mathrm{kcal} / \mathrm{mol}$ for the HBD probes). This process improved the representation of the binding hotspot defined by the PhF. In addition, the radius of gyration was calculated and used as a guide to determine the radius of the PhFs $(0.4-2.5 \AA)$.

As a control the PhFs identified during MIF analysis were compared with known PhFs derived from published crystal structures with bound inhibitors and two PhFs were found to have moved $\sim 2 \AA$ within their respective binding pockets. These $\mathrm{PhFs}$ represent the aminopropyl nitrogen group of dcAdoMet and the nitrogen of 4MCHA (corresponding to the non-attacking nitrogen of putrescine). Inspection of two crystal structures [PDB:2I7C] and [PDB:2PT9] revealed the interaction of two solvent molecules with chemical moieties binding within these PhFs. The coordinate positions for these water molecules were determined using a water probe and subsequently added to the structures and energy optimized prior to MIF analysis.

EVs i.e. atoms within $7 \AA$ of AdoDATO co-crystallized with PfSpdS [PDB:2I7C], were specified to facilitate database searching. The atomic coordinates of the selected EVs within the sub-ensemble of structures were extracted and their centres of masses were calculated to incorporate target flexibility (in-house python script). These 'dynamic' EVs are improved representations of the boundaries of the active site since they indicate an average position of the atoms over time. The EVs were overlaid with the co-crystallized ligands AdoDATO, dcAdoMet and 4MCHA to identify steric clashes, which were subsequently removed.

\section{Pharmacophore model selection}

The active site of SpdS contains binding cavities for dcAdoMet and putrescine [50,51]. The putrescine binding cavity of PfSpdS also accommodates the aminopropyl chain of spermidine at its non-attacking end when spermine is synthesized [18,20] (Figure 2). Figure 3 shows the subdivision of the $r$-shaped active site into four binding regions with the native substrates within their respective binding cavities for dynamic pharmacophore models (DPM1 to DPM4) to facilitate pharmacophore searches and explore specific regions within the active site. The DPM1 binding region was selected to explore the putrescinebinding cavity; the DPM2 binding region was selected to explore compounds that protrude from the putrescinebinding cavity into the dcAdoMet cavity; the DPM3 binding region was selected to explore compounds that protrude from the dcAdoMet-binding cavity into the putrescine binding cavity; while the DPM4 binding region was used to explore the entire active site (Figure 3).

\section{DPM1 binding cavity}

Two PhFs that best described the receptor-derived pharmacophore model for 4MCHA were used to verify the model's ability to identify this inhibitor. The basic model showed a best-fit value of $0.98 / 2$ (best-fit value/ number of PhFs; Figure 1) and thus confirms that 4MCHA would have been identified by screening if it was present in the virtual chemical library. The selected PhFs (with a bias towards the PfSpdS structure cocrystallized with dcAdoMet and 4MCHA, [PDB:2PT9]) derived from the MIF analyses were used to construct a shortlist of eight DPMs consisting of either three or four $\mathrm{PhFs}$ each. These eight DPMs were screened against the drug-like subset of the ZINC database and between 100 


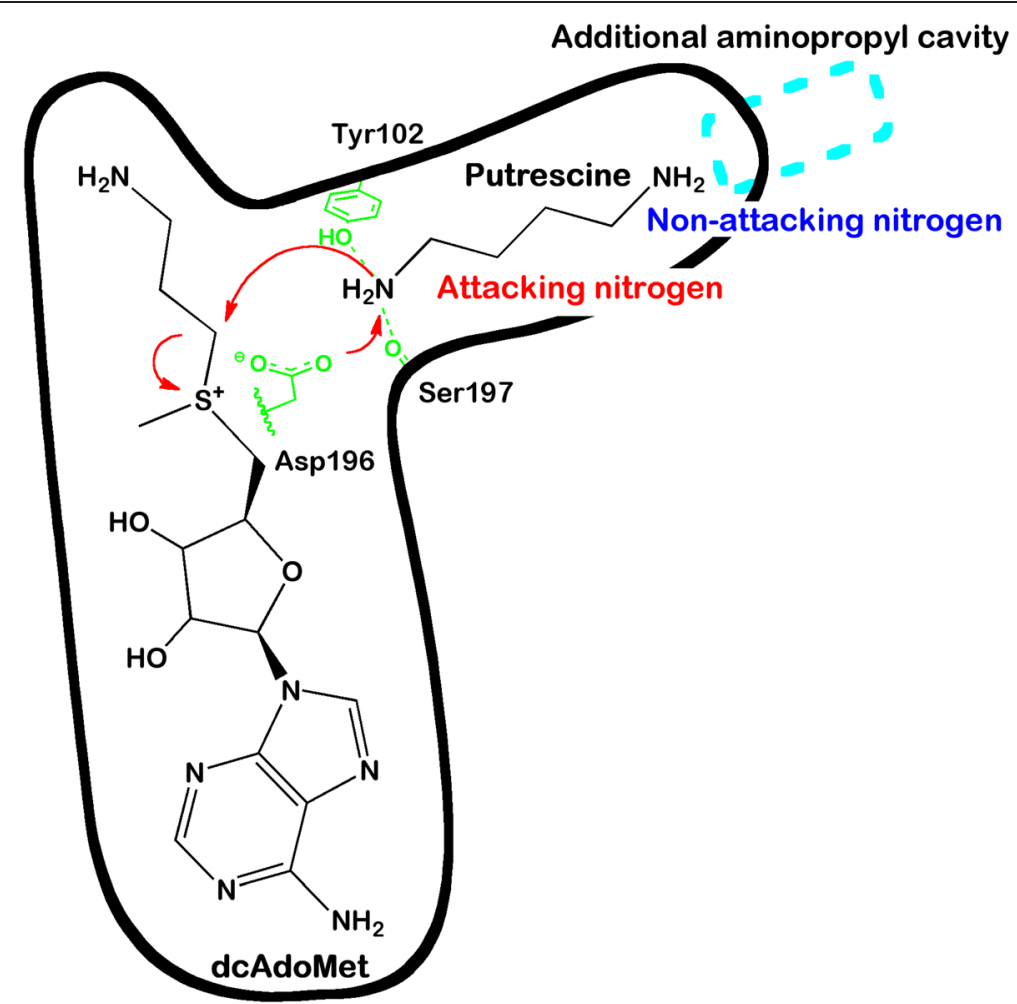

Figure 2 A representation of putrescine and dcAdoMet within their binding cavities. Residues important for catalysis are shown in green. Red arrows indicate the proposed attack by the putrescine amino group (attacking nitrogen) on the methylene carbon of the aminopropyl chain of dcAdoMet resulting in the catalysis that produces spermidine via a $\mathrm{SN}_{2}$ reaction. Highlighted in cyan is the aminopropyl cavity (in the vicinity of the non-attacking nitrogen of putrescine) that can be accommodated by the aminopropyl chain of spermidine to catalyze the production of spermine.

and 22,000 hits were identified. Eleven compounds were selected following visual inspection and filtering based on best-fit values and molecular mass. Docking was used to further filter and rank the compounds based on their binding poses and docking energies within the active site.

Similar to 4MCHA, 1-(3-ammoniopropyl)-4-methylpiperidin-1-ium (compound 1) satisfied both the HYD and positive ionizable (PI) PhFs with an additional aminopropyl chain that fits to a novel PI PhF identified within the non-attacking region of the putrescine-binding cavity (Figure 1). Compound 1 showed a reasonable best-fit value of $0.53 / 3$ with a docking energy of $-7.4 \mathrm{kcal} / \mathrm{mol}$ and reduced PfSpdS activity by $17.0 \pm$ $9.4 \%$ at $100 \mu \mathrm{M}$ (Table 1). A binding pose where the aminopropyl chain of compound 1 traverses the catalytic centre and binds in the dcAdoMet cavity was predicted to be less favourable but could not be excluded.

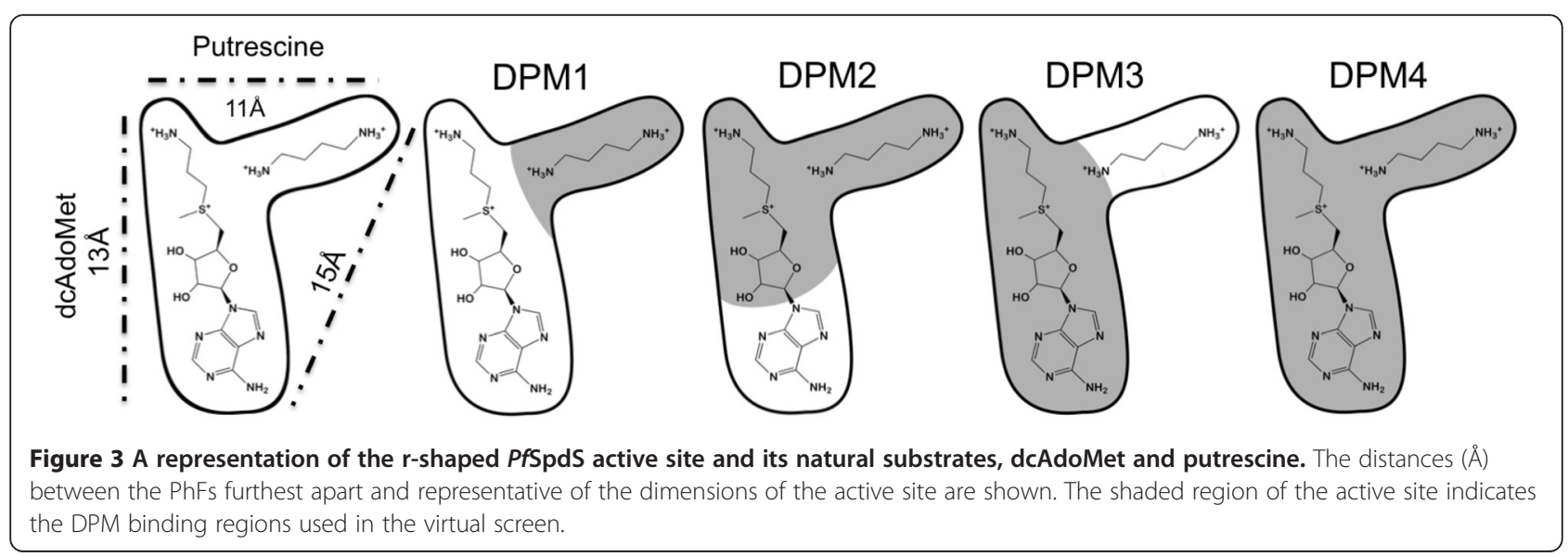


Many of the hits obtained from the virtual screen against PfSpdS contained either a piperidine or piperazine ring and it was therefore of interest to determine whether these rings would be able to fit into the same chemical space as the cyclohexane ring of $4 \mathrm{MCHA}$. A similarity search identified a $4 \mathrm{MCHA}$ analogue, trans4-amino-1-methylpiperidine (compound 2; Figure 1), which differs from $4 \mathrm{MCHA}$ by a ring nitrogen. Docking of compound 2 with its ring nitrogen in the protonated state showed a similar orientation to $4 \mathrm{MCHA}$ with regards to its methyl and amino group (docking score $-8.2 \mathrm{kcal} / \mathrm{mol} ; \mathrm{pK}_{\mathrm{a}}$ of $N$-methylpiperidine 10.1) [52]. However, compound 2 reduced PfSpdS specific activity by only $6.9 \pm 5.8 \%$ at $100 \mu \mathrm{M}$ (Table 1 ) suggesting that piperidine rings are not well accommodated within the putrescine-binding cavity.

\section{DPM2 binding cavity}

Fourteen DPMs were generated from the DPM2 binding cavity for virtual screening. The PhFs for this region were selected from both the sub-ensemble of structures and crystallographic data. PhFs selected to represent the non-attacking putrescine-binding region were biased to the data derived from PfSpdS co-crystallized with 4MCHA and dcAdoMet. The virtual screens of the 14 DPMs resulted in between one and 1,800 hits for the respective DPMs, from which 24 compounds were selected for docking. Two compounds were chosen for in vitro testing (Additional file 1). (5S)-6-amino-5-[[(2S)2-azaniumyl-3-(4-hydroxyphenyl) propanoyl] amino]-6oxohexyl] azanium (compound 3) was selected based on its good best-fit value (0.91/4) and favourable docking energy $\quad(-12.5 \mathrm{kcal} / \mathrm{mol})$. 3-[4-(3-azaniumylpropyl)piperazine-1,4-diium-1-yl]propylazanium (compound 4) showed a low best-fit value $(0.15 / 4)$ but a docking score of $-9.8 \mathrm{kcal} / \mathrm{mol}$ and a good binding pose as revealed by visual inspection. Compound 4 contains a piperazine ring and was predicted to bind within the non-attacking nitrogen binding site of putrescine. The other aminopropyl chain of compound 4 was predicted to bind in the same binding pocket as the aminopropyl chain of dcAdoMet. However, in vitro data showed that compounds 3 and 4 did not inhibit the enzyme at a $100 \mu \mathrm{M}$ concentration (Additional file 1).

\section{DPM3 and DPM4 binding cavities}

Ten different DPMs were constructed for the DPM3 cavity each consisting of four to six PhFs and between 0 to 1,813 hits were identified by virtual screening. Filtering and visual inspection identified seven compounds for docking. $N^{\prime}$-[(2-hydroxy-4-oxocyclohexa2,5-dien-1-ylidene)methyl]-3-(4-methylpiperazine-1,4diium-1-yl)-propanehydrazide (compound 5) and [(2R)-3-azaniumyl-2-hydroxypropyl]-[(2S)-2-hydroxy- 3-piperidin-1-ium-1-ylpropyl]-methylazanium (compound 6) represent new scaffolds for binding to the dcAdoMet cavity and were selected for in vitro testing. However, neither compound showed reduction in PfSpdS activity (Additional file 1).

The DPM4 cavity covers the entire active site and eight DPMs consisting of four or five PhFs each, were prepared for screening. These DPMs defined AdoDATO with a best-fit value of $0.66 / 5$ and served as a control to validate that known inhibitors of this binding cavity would have been identified. Screening resulted in 0 to 80 hits for the eight DPMs from which six compounds were selected for docking. Only [(2S)-6-azaniumyl-1[[(2S)-6-azaniumyl-1-(naphthalen-2-ylamino)-1-oxohexan2-yl]amino]-1-oxohexan-2-yl]azanium (compound 7) occupied the entire cavity but showed a low best-fit value of $0.14 / 5$ with a docking energy of $-10.5 \mathrm{kcal} / \mathrm{mol}$ and showed no inhibition (Additional file 1).

\section{Knowledge-based design of inhibitors}

It was postulated that the lower $\mathrm{IC}_{50}$ of adenosylspermidine compared to AdoDATO $[21,38]$ against mammalian SpdS is due to additional hydrogen bonding in the catalytic centre via the secondary amine of adenosylspermidine, which mimics the attacking nitrogen of putrescine. Binding free energy $(\Delta G)$ calculations of adenosylspermidine and an analogue, in which the nitrogen atom is replaced by a carbon atom, showed an increase of $15 \mathrm{kcal} / \mathrm{mol}$ for the latter (Figure 1; arrow). Conversely, replacing the corresponding carbon atom in AdoDATO by nitrogen reduced the binding free energy of the analogue by $35 \mathrm{kcal} / \mathrm{mol}$ (MM-GB/SA; Schrödinger; Figure 1; arrow).

Using the above information, the DPM2 cavity was selected as a basis for the knowledge-based design of inhibitors since it includes the catalytic centre, the putrescine binding cavity and part of the dcAdoMet binding pocket (Figure 3). Preference was therefore given to small molecules able to favourably cross the electronrich catalytic centre between the binding cavities (from the putrescine binding cavity to the dcAdoMet cavity), which contain an amine group and would promote closure of the gate-keeping loop in the presence of MTA. Knowledge-based design required compounds to match the chemical features of important PhFs that describe the DPM2 binding cavity and included the prominent PI $\mathrm{PhF}$ that represents the aminopropyl chain of dcAdoMet and the non-attacking nitrogen of putrescine (Figure 4A). In addition, the PI PhF that describes the attacking nitrogen of putrescine and the HYD PhF of putrescine's butyl chain, were also included (Figure 4A).

$N$-(3-aminopropyl)-trans-cyclohexane-1,4-diamine (compound 8) was derived from the above considerations and represents a basic scaffold for the DPM2 cavity (Figure 4B). Docking of compound 8 showed the expected binding 


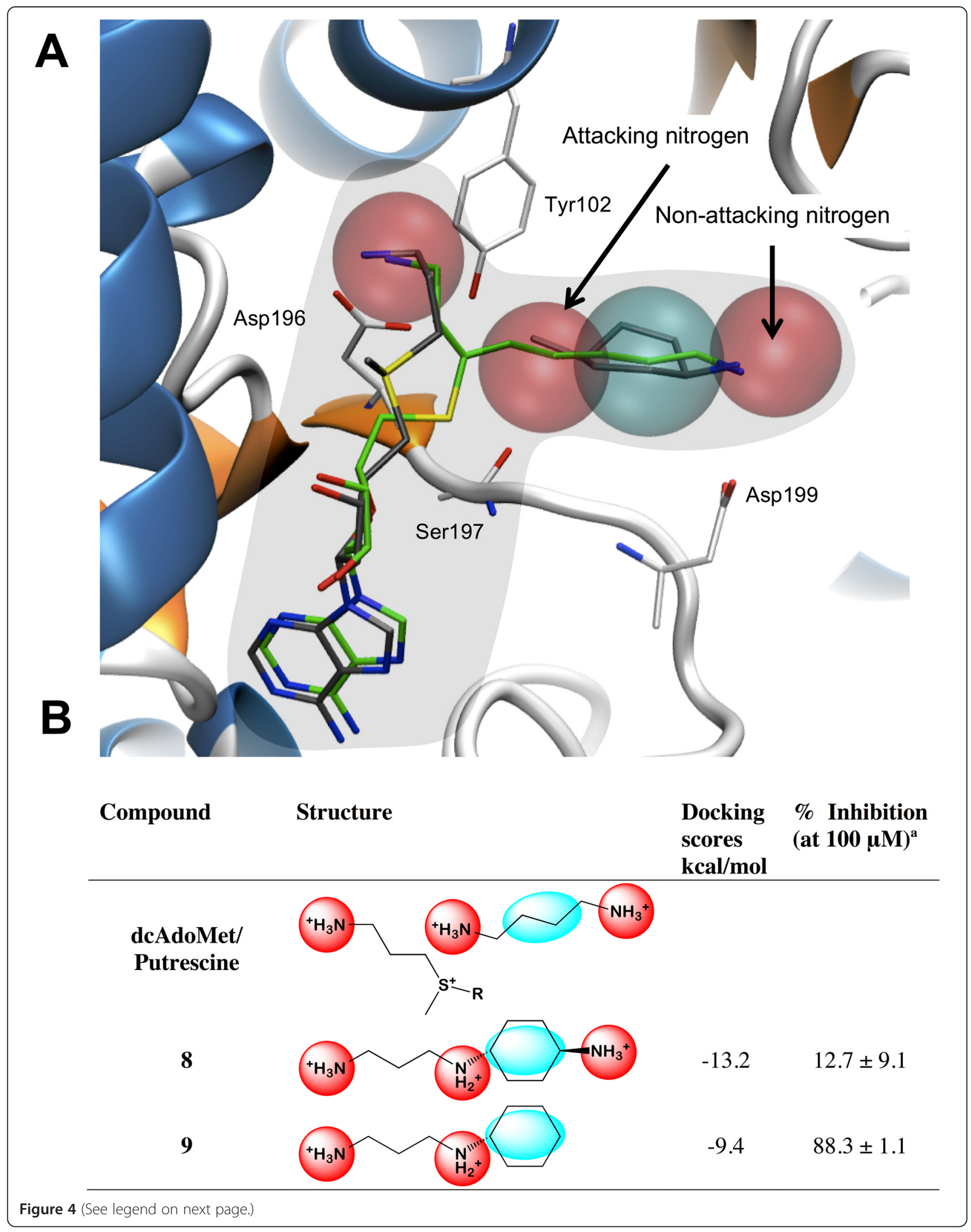


(See figure on previous page.)

Figure 4 The most important binding characteristics of the DPM2 cavity and the compounds selected to explore this region. (A) The PhFs best describing the binding characteristics of the DPM2 cavity. The red and blue spheres represent the PI and hydrophobic features, respectively. The PhFs of AdoDATO are shown in green while those of 4MCHA and dcAdoMet are in grey. The amino acid residues in white represent those that best define the interactions described by the PhFs. From left to right the red spheres represent the PI features of the aminopropyl chain of dcAdoMet, the middle feature represents the attacking nitrogen of putrescine and on the right is the feature of the non-attacking nitrogen of putrescine. In blue is the hydrophobic feature representative of the butyl group of putrescine. (B) The structures of the compounds including their docking scores, selected for inhibition assays of PfSpdS activity at $100 \mu \mathrm{M}$. ${ }^{a}$ Results represent the percentage PfSpdS activity compared to untreated enzyme of the three independent experiments performed in duplicate. Data are given as \pm SEMS, $n=3$ or $n=4$.

poses with a binding energy of $-13.2 \mathrm{kcal} / \mathrm{mol}$ (Figure $4 \mathrm{~B}$ ). The cyclohexylamine moiety is predicted to bind similarly to 4MCHA with the corresponding amino group forming a hydrogen bond with Asp199 (gate-keeping loop residue). The bridging nitrogen connecting the aminopropyl chain of compound 8 to the cyclohexane ring is predicted to form two hydrogen bonds; one with Tyr102 (residue from Rossman-like fold) and another with either the backbone carbonyl group of Ser197 (gate-keeping loop residue) or interchangeably with Gln93. These hydrogen bonds are expected to reduce the binding penalty of an aliphatic carbon chain that bridges the catalytic centre as suggested by the calculations of the $\Delta$ G's for AdoDATO and adenosylspermidine and their analogues (see above). The in silico model also predicted that compound 8 would cooperatively bind with MTA to facilitate closure of the gate-keeping loop. This compound reduced PfSpdS activity by only $12.7 \pm$ $9.1 \%$ at $100 \mu \mathrm{M}(\mathrm{n}=3$; Figure $4 \mathrm{~B})$ with an $\mathrm{IC}_{50}$ value of $619 \pm 144 \mu \mathrm{M}(\mathrm{n}=3$; Table 1).

In addition, $N$-(3-aminopropyl)-cyclohexylamine (compound 9) was identified as being similar to compound 8 differing by missing a 4-amino group (similarity search, SciFinder; Figure $4 \mathrm{~B})$. This compound was therefore expected to assume the same binding pose and hydrogen bond pattern as compound 8 (Figure 4B). Docking of compound 9 showed a good binding pose and a binding energy of $-9.4 \mathrm{kcal} / \mathrm{mol}$ (Figure 4B). In vitro testing of compound 9 at $100 \mu \mathrm{M}$ showed an $88.3 \pm 1.2 \%(\mathrm{n}=5)$ reduction in PfSpdS activity (Figure 4B) with an $\mathrm{IC}_{50}$ value of $7.4 \pm 2.4 \mu \mathrm{M}(\mathrm{n}=3$; Table 1$)$. This value is similar to the $\mathrm{IC}_{50}$ values of AdoDATO [20] and 5-amino-1pentene (APE) [18] but higher than 4MHCA (Table 1).

\section{X-ray crystallography}

To experimentally determine the binding mode of compounds 8 and 9 crystallization screens were performed with a 39 amino acid N-terminal truncated PfSpdS protein as described by Dufe et al. [20]. The purified protein was mixed with either compound 8 or 9 in the presence or absence of MTA to produce PfSpdS-inhibitor-MTA or PfSpdS-inhibitor complexes, respectively. Testing of numerous conditions similar to the ones previously described by Dufe et al. [20] for crystallization of PfSpdS, yielded crystals within several days that diffracted to $1.9 \AA$ (Figure 5). Data collection and refinement statistics for the compound 8 and MTA PfSpdS crystal
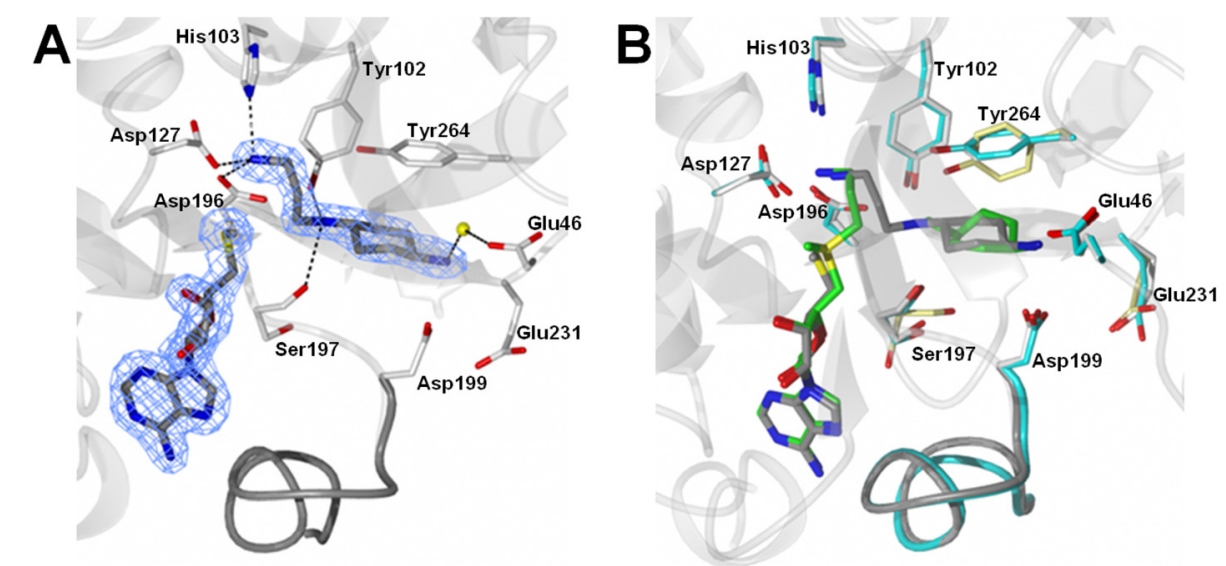

Figure 5 PfSpdS co-crystallized with MTA and compound 8. (A) The electron densities of MTA and compound 8 are shown in blue ( $\sigma=1)$. The residues involved in hydrogen bonding with compound 8 are indicated with dotted lines. The gate-keeping loop covering the active site is shown as a grey ribbon with two of its residues, Ser197 and Asp199, which together with residues Glu46, Tyr102, His103, Asp127 Asp196, Glu231 and Tyr264, forms the active site. The solvent molecule involved in an interaction with compound 8 via Glu46 is shown as a yellow sphere. (B) MTA and compound 8 of the 3RIE structure in grey are superimposed with 4MCHA and dcAdoMet in green from the 2PT9 crystal structure. The active site residues involved in compound 8 and $4 \mathrm{MCHA}$ binding are shown in grey and cyan, respectively. 
structure are listed in Table 3. As with all the previous structures of PfSpdS, the protein crystallized in space group $C 121$ with three molecules in the asymmetric unit (Table 3). Subunits B and C form a homodimer with a buried interface of $1424 \AA^{2}$ as analysed with PISA [53]. The PfSpdS structure of MTA with compound 8 [PDB:3RIE] superimposes with RMSD values of 0.31 and $0.33 \AA^{2}$ with the ligand free [PDB:2PSS] and dcAdoMet4MCHA [PDB:2PT9] structures, respectively (Figure 4B). PfSpdS with compound 8 and MTA showed high occupancies for both ligands in all the protein molecules in the asymmetric unit. MTA is bound in the dcAdoMet binding site of PfSpdS structures as previously shown ([PDB:2HTE]; [PDB:3BP7]). The binding pose of compound 8 confirmed the in silico predicted binding orientation of the compound within the DPM2 binding site in the presence of MTA as shown in Figures 4A and 6A.

\section{Table 3 Crystallography data collection and refinements} statistics

\begin{tabular}{|c|c|}
\hline \multicolumn{2}{|l|}{ Data collection } \\
\hline Beamline & MAX II I911-2 \\
\hline Wavelength $(\AA)$ & 1.04 \\
\hline Space group & $C 121$ \\
\hline Unit cell $(a ; b ; c ; \beta)(\AA)$ & $196.8 ; 134.6 ; 48.5 ; 94.55^{\circ}$ \\
\hline Molecules per ASU & 3 \\
\hline Resolution ( $\AA$ ) & $20.0-1.9(1.95-1.9)$ \\
\hline Observed reflections & $413845(30068)$ \\
\hline Unique reflections & 98147 (7216) \\
\hline Completeness (\%) & $99.8(100)$ \\
\hline Multiplicity & $4.2(4.2)$ \\
\hline$</|>|<\sigma\rangle$ & $18.47(3.92)$ \\
\hline$R_{\text {meas }}(\%)$ & $5.9(41.1)$ \\
\hline \multicolumn{2}{|l|}{ Refinement statistics } \\
\hline Resolution & $20-1.9(1.94-1.89)$ \\
\hline Reflections & $93239(5053)$ \\
\hline$R_{\text {cryst }}(\%)$ & $17.8(29.6)$ \\
\hline$R_{\text {free }}(\%)$ & $21.0(30 / 7)$ \\
\hline Atoms & 7493 \\
\hline Non-hydrogen & 6876 \\
\hline Water & 617 \\
\hline Average B-factor $\left(\AA^{2}\right)$ & 25.46 \\
\hline \multicolumn{2}{|l|}{ RMSD from ideal } \\
\hline Bond lengths $(\AA)$ & 0.029 \\
\hline Bond angles $\left(^{\circ}\right)$ & 2.1 \\
\hline \multicolumn{2}{|c|}{ Ramachandran statistics $(\%)^{a}$} \\
\hline Favoured & 97.01 \\
\hline Outliers & 0.12 \\
\hline
\end{tabular}

Values in parenthesis are for the high resolution shell.

${ }^{a}$ Ramachandran statistics were calculated using Molprobity [47].
The non-attacking amine of the cyclohexane ring is hydrogen-bonded to Glu46 via a water molecule and to the side chain of Asp199. The bridging amino group is within hydrogen bonding distance of Tyr102 and Ser197 and facilitates the traversal of compound 8 across the catalytic centre. The nitrogen (epsilon) of His103 and one carboxyl oxygen each of Asp127 and Asp196 do form hydrogen bonds to the terminal aminopropyl nitrogen of compound 8 (Figure 6A). These interactions correspond to the interactions of the aminopropyl nitrogen of dcAdoMet in complex with 4MCHA. Moreover, clear electron density for the gate-keeping loop was observed when both compound 8 and MTA were present. The average $\mathrm{B}$ factor values for the loop residues are in the range of the overall B factors (19.30/25.46 overall/loop) and comparable with that of the structure containing AdoDATO ([PDB:2I7C]; Additional file 4). In the presence of both dcAdoMet and 4MCHA ([PDB:2PT9]; Additional file 4) the gate-keeping loop of the resolved structure also has high B values [20]. Previous studies have shown that the gate-keeping loop is disordered in the ligand free structure [PDB:2PSS] or when only MTA is present [PDB:2HTE]. Crystallization trials with compound 9 and MTA resulted in crystals with poor diffraction quality and weak density and could not be used for structure solving.

\section{Proposed alternative binding poses for compounds 8 and 9}

The crystal structure with PfSpdS in complex with MTA and compound 8 confirmed the in silico predicted binding poses of these two ligands in the active site. Since no resolved crystal structure of PfSpdS with bound MTA and compound 9 could be obtained, there is no structural explanation for the unexpected discrepancy in the $\mathrm{IC}_{50}$ values between compounds 8 and 9. However, Shirahata et al. identified compound 9 as an inhibitor of human spermine synthase but not of human SpdS [22]. PfSpdS also accepts spermidine as secondary substrate to synthesize spermine and Shirahata's results therefore suggest that compounds 8 and 9 bind in the active site in a reversed orientation, i.e., aminopropyl chains facing the non-attacking side of putrescine binding cavity, with dcAdoMet present instead of MTA (Figure 6). The implication of this mode of binding by compounds 8 and 9 is that these ligands must compete with both putrescine and spermidine for binding in presence of dcAdoMet, which is supported by preliminary kinetic experiments showing a mixed-type competitive inhibition between compound 9 and putrescine $\left(\mathrm{K}_{i}\right.$ of $2.8 \mu \mathrm{M}$; Additional file 5). A schematic representation of the binding poses of compounds 8 and 9 in PfSpdS (in the presence of dcAdoMet) relative to those of spermidine as substrate and product as well as spermine is presented in Figure 7. 


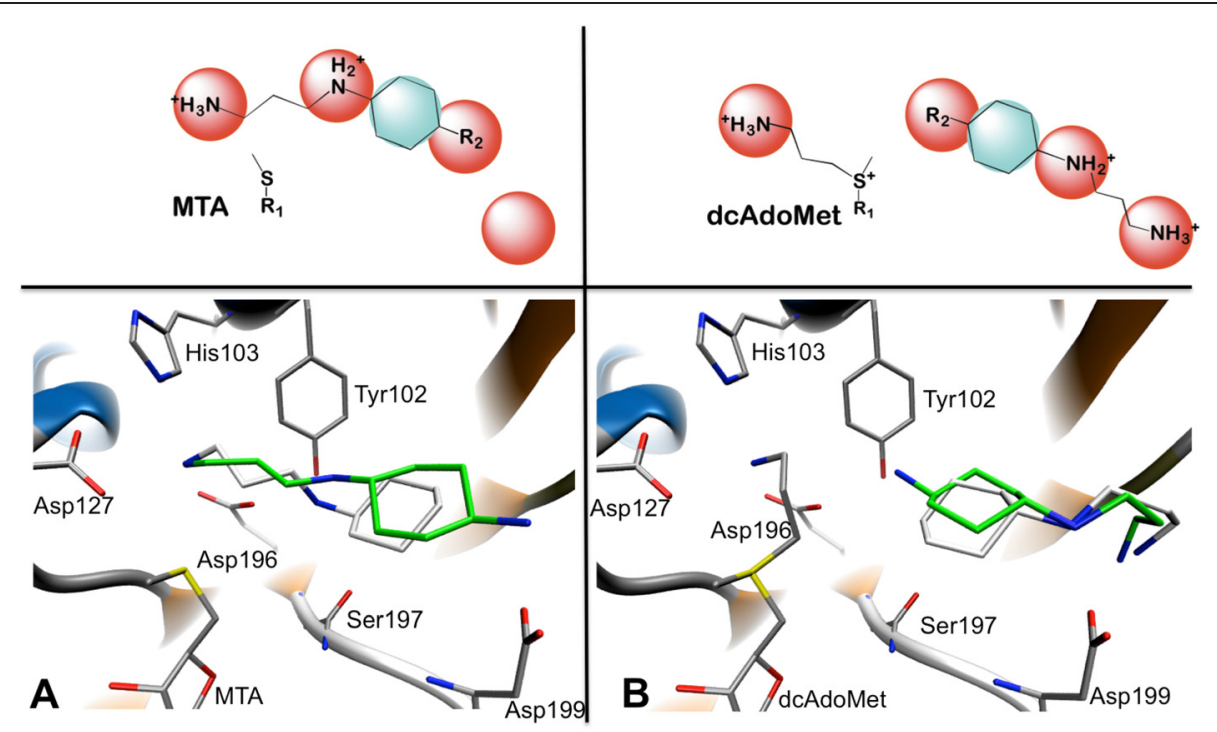

Figure 6 Different binding poses that compounds 8 (green) and 9 (white) can assume upon binding with MTA and dcAdoMet, respectively. (A) Compounds 8 and 9 traverse the catalytic centre when cooperatively bound with MTA. The aminopropyl chains of compounds 8 and 9 bind in the chemical space that are normally occupied by the aminopropyl chain of dcAdoMet. (B) Compound 8 and 9 assume a reverse binding pose in the presence of dcAdoMet. Compounds 8 and 9 form hydrogen bonds with Asp199 and a water molecule anchored by lle92 and Glu46 (not shown) in the non-attacking end of the putrescine-binding cavity. An additional hydrogen bond is formed by compound 9 with Tyr102.

Docking of compound 9 in the reverse orientation with dcAdoMet instead of MTA furthermore showed it to be the stronger binder $(-8.5 \mathrm{kcal} / \mathrm{mol})$ of PfSpdS (Figure 6) than compound $8(-7.1 \mathrm{kcal} / \mathrm{mol})$. Most notable is the increase in the predicted binding penalty energy due to strain between the receptor in the complex and the receptor in the free state for compound 8 in comparison to compound $9(11.3 \mathrm{kcal} / \mathrm{mol}$ and $3.8 \mathrm{kcal} / \mathrm{mol}$ respectively, using MM-GB/SA). These results would explain the significant differences in the $\mathrm{IC}_{50}$ values of compounds 8 and 9 . In addition, compound 8 bound in the reverse orientation, i.e., with the primary amine of the cyclohexyl ring facing the catalytic centre, also acts as a pseudo-substrate for PfSpdS (JS et al., unpublished results). This in effect would lower its concentration as catalysis proceeds.

Attempts to grow diffraction quality PfSpdS crystals with bound compound 9 and dcAdoMet were unsuccessful. Against this background further insight into the putrescine/spermidine binding pocket was obtained from the PfSpdS structure co-crystallized with spermidine [PDB:2PWP] and spermine [PDB:3B7P]. Compound 8 assumes a similar binding orientation [PDB:3RIE] to that of spermidine in the presence of MTA [PDB:4CXM] with its aminopropyl chain also located in the dcAdoMet cavity and similar interactions with the catalytic centre. Interestingly, one of the aminopropyl chains of spermine co-crystallized with MTA [PDB:3B7P] is located in the
dcAdoMet binding cavity and its putrescine moiety located in the putrescine binding cavity similar to spermidine co-crystallized with PfSpdS [PDB:2PWP]. The other or second aminopropyl chain of spermine is located in the non-attacking end of the putrescine-binding cavity and show two distinct binding poses in the three different protein chains. The aminopropyl chains of compounds 8 and 9 when bound in the reverse orientation are similar to the dominating binding pose of spermine noticed in two of the three subunits [PDB:3B7P] (Additional files 6 and 7). Noteworthy, two residues Glu231 and His236, showed altered conformations that facilitate the accommodation of the aminopropyl chain of spermine in the non-attacking end of the putrescine-binding cavity.

Finally, a comparison between the proposed binding poses of compounds 8 and 9 in PfSpdS to that of spermine crystallized with $H s S p m S$ demonstrated distinct binding cavities for the aminopropyl chains in the non-attacking end of the putrescine/spermidine binding cavity (Additional files 6 and 7). Docking of compounds 8 and $9(-8.8$ and $-8.7 \mathrm{kcal} / \mathrm{mol}$, respectively) to $H s \mathrm{SpmS}$ [PDB:3C6K] showed that their aminopropyl chains bind in the same orientation and cavity to that of the enzyme co-crystallized with spermidine. These observations suggest that compounds may be designed with selectivity for PfSpdS and not $H s S p m S$ by exploring the extra aminopropyl cavity that allows PfSpdS to accommodate spermidine for synthesis of spermine. 


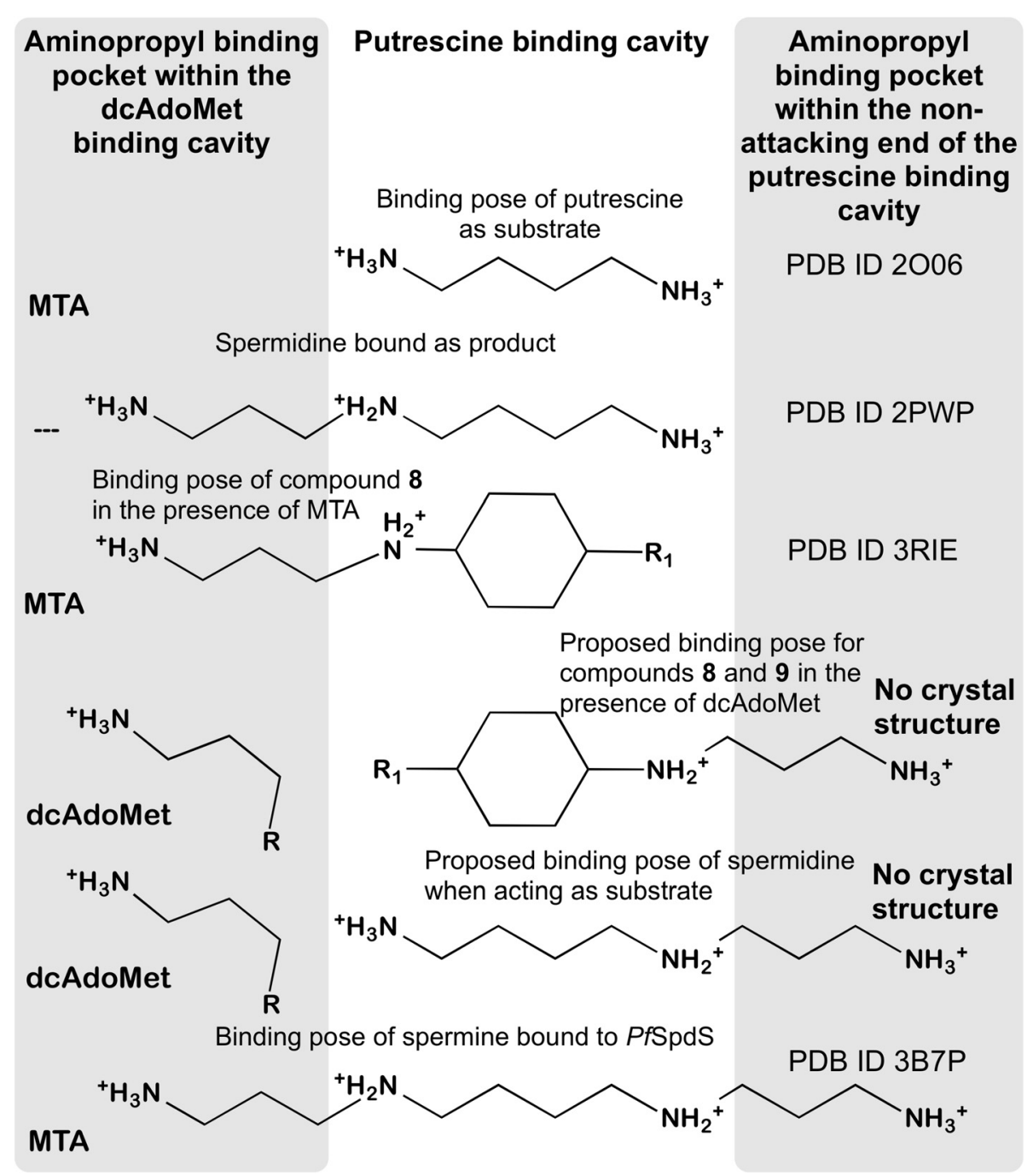

Figure 7 A schematic representation of the binding poses of putrescine spermidine, spermine, compound 8 and compound 9 to SpdS. The grey highlighted columns depict the two different binding pockets for the aminopropyl chains of spermidine, spermine, compound 8 and compound 9 . The grey column on the left depicts the aminopropyl binding pocket associated with the dcAdoMet binding cavity. The grey column on the right depicts the additional aminopropyl binding pocket located in the non-attacking end of the putrescine binding cavity responsible for the ability of PFSpdS to produce spermine. The white column depicts the putrescine binding cavity and the corresponding moieties of the analogues depicted in this Figure. PDB IDs are given when the binding poses of compounds were from crystal structures. When ligands are resolved in the presence of MTA or dcAdoMet they are indicated in the aminopropyl binding pocket column of dcAdoMet (grey column on the left). For compounds resolved with dcAdoMet their aminopropyl chains are displayed in their binding cavities. $R_{1}=\mathrm{NH}_{3}^{+}$is compound 8; $\mathrm{R}_{1}=\mathrm{H}$ is compound 9.

\section{Discussion}

The most effective inhibitors for SpdS were derived following a ligand-based approach and include 4MCHA, AdoDATO and adenosylspermidine. 4MCHA is the most potent inhibitor of PfSpdS with an $\mathrm{IC}_{50}$ value of $1.4 \mu \mathrm{M}$ followed by AdoDATO with an $\mathrm{IC}_{50}$ value of $8.5 \mu \mathrm{M}$ (Table 1) $[18,20]$. The 170 -fold lower $\mathrm{IC}_{50}$ value of AdoDATO for mammalian SpdS compared to the $P$. falciparum enzyme has been suggested to be due to a higher $K_{m}$ value of the latter enzyme for dcAdoMet
(35 $\mu \mathrm{M})$ compared to the mammalian enzyme $(7-25 \mu \mathrm{M})$ $[18,20,38]$. The active site of PfSpdS accommodates dcAdoMet and either putrescine or spermidine in the second substrate-binding cavity. The latter substrate is converted into spermine and accounts for $15 \%$ of the enzyme's activity [18]. In addition, binding of ligands into the putrescine binding cavity is suggested to occur subsequent to binding of ligands into the dcAdoMet binding cavity [20]. In the first structure-based drug design study for PfSpdS, AdoDATO was used to select PhFs for the generation of 
pharmacophore models [23]. Screening of the models against an in-house chemical library identified several binders to the active site but did not yield any promising inhibitory compounds. Re-evaluation of the active site suggested that its size and shape are unfavourable for a standard virtual screen and was, therefore, divided in this study into four binding regions as shown in Figure 3. 'Dynamic' receptor-based pharmacophore models (DPM1-4; Figure 3) were developed for each region and information obtained from previous studies as well as new information derived in this study was incorporated [22,54]. The methodology used here uniquely also included protein flexibility, which was captured during phase space sampling and represented as a sub-ensemble of structures. Importantly, this approach highlighted a conformational change in Gln229 between the ligand free- and ligand-bound states of PfSpdS, which significantly alters the binding characteristics of the putrescine-binding cavity (Additional file 3 ). Potential inhibitors were identified from the derived pharmacophore models by a combination of virtual screening of the ZINC database, knowledge-based design and similarity searches. Compounds that matched features of interest were subsequently docked into the PfSpdS active site before being selected for inhibition assays.

By exploring the DPM1 cavity compound 1 (Figure 1) was identified and predicted to bind with its aminopropyl chain protruding into the non-attacking side of the putrescine-binding cavity and the piperidine ring overlapping with the cyclohexane ring of 4MCHA. The possibility that it binds in a reversed orientation as an explanation for the weak inhibition of PfSpdS at $100 \mu \mathrm{M}$, was excluded due to unfavourable docking scores. This result suggested that a piperidine ring is not easily accommodated within the DPM1 cavity when the ring nitrogen faces the catalytic centre and is supported by results obtained for compound 2 .

No noteworthy inhibitory compounds were identified by virtual screening of the binding cavities of DPM2-4. The result for the DPM4 binding cavity was however not surprising considering the dimensions and shape of the binding cavities described by the pharmacophore models (Figure 3) and the limited chemical space covered by the drug-like subset of the ZINC database.

The gate-keeping loop (residues 197-205) that covers the active site has been proposed to play an important role in putrescine-binding as well as inhibition of SpdS by $4 \mathrm{MCHA}[18,50]$. Putrescine forms hydrogen bonds with loop residues Ser197 and Asp199 at the attacking and non-attacking ends of the putrescine-binding cavity of PfSpdS, respectively. 4MCHA binds in the putrescine cavity by hydrogen bonding to loop residue Asp199 but does not form hydrogen bonds at the catalytic centre. It was therefore postulated that compounds that traverse the catalytic centre and bind to the gate-keeping loop at more than one site would result in an extended closed state of the loop and an improved inhibitor of PfSpdS compared to 4MCHA. The SpdS inhibitor AdoDATO is a 3-7-fold less efficient inhibitor of mammalian SpdS than adenosylspermidine (Table 1) [21,38]. The aliphatic aminopentyl chain of both multi-substrate analogues traverses the electron rich catalytic centre and interacts with loop residue Asp199 at the non-attacking end of putrescine, similar to 4MCHA. Binding free energy calculations here show that the lower $\mathrm{IC}_{50}$ value of adenosylspermidine compared to AdoDATO is most likely due to the secondary amine in the aliphatic aminopentyl chain of adenosylspermidine (Figure 1; arrow), which permits additional hydrogen bonding with Ser197 (gate-keeping loop) and Tyr102. Binding free energy calculations performed on PfSpdS showed similar results.

Prompted by the above observations, small molecule inhibitors that directly interact with the gate-keeping loop were knowledge-based designed, based on four PhFs identified in the DPM2 binding cavity (Figure 4A). Compounds 8 and 9 were predicted to assume binding poses in which they cross the catalytic centre with their aminopropyl chains bound within the dcAdoMet-binding cavity provided that MTA is present (Figure 6A). This was confirmed for compound 8 by a crystal structure co-crystallized with MTA (Figure 5) with similar B factor values for the gate-keeping loop to those of the PfSpdS crystal structure resolved with AdoDATO (Additional file 4).

It was envisioned that once dcAdoMet is converted into MTA, binding of compounds 8 and 9 would be promoted, especially of compound 8 due to additional hydrogen bond formation with the gate-keeping loop, which would prolong its closure. However, the 100-fold weaker $\mathrm{IC}_{50}$ of compound 8 compared to compound 9 does not support this novel hypothesis. Pre-incubation of PfSpdS with MTA up to concentrations of $100 \mu \mathrm{M}$ prior to assays furthermore did not change the inhibitory capacity of these compounds (AIL et al. unpublished results). Preliminary kinetic experiments with PfSpdS however indicated a mixed-type competitive inhibition between compound 9 and putrescine with a $K_{i}$ of $2.8 \mu \mathrm{M}$ (Additional file 5). Shirahata et al. [22] identified compound 9 as an inhibitor of mammalian spermine synthase, which however did not inhibit mammalian spermidine synthase. A plausible explanation for the results presented here, therefore, is that competition between the aminopropyl chains of compounds 8 and 9 with that of dcAdoMet (Figures 5 and 6) may force their binding in a reversed orientation with their chains now facing the non-attacking end of the putrescine binding cavity, i.e., acting as spermidine analogues. Docking results with dcAdoMet instead of MTA indicated a higher binding affinity for compound 
9 compared to compound 8 when their binding orientation was reversed (Figures 6 and 7). Moreover, a three-fold increase in receptor (PfSpdS) strain was predicted for compound 8 in comparison to compound 9 . Taken together, these results would explain the observed disparity between the $\mathrm{IC}_{50}$ values of compounds 8 and 9. An earlier report has shown that the diamine substrate, trans-1,4-diaminocyclohexane, acts as a pseudosubstrate $(\sim 12 \%)$ for mammalian SpdS [22]. Similar results were found for compound 8 (JS et al., unpublished results), which in consequence would lower its effective inhibitory capacity as catalysis proceeds since it accepts an aminopropyl chain from dcAdoMet, which diminishes its residence time within the binding cavity. Importantly, no evidence was found for compound 9 acting as a pseudo-substrate. It is accepted that some percentage of compounds 8 and 9 may also bind with their chains facing the catalytic centre, albeit briefly as catalysis proceeds and MTA is produced.

Interestingly, a comparison of the $H s \mathrm{SpmS}$ [PDB:3C6M] and PfSpdS [PDB:3B7P] structures resolved with spermine show two distinct binding pockets for the aminopropyl chain of spermine at the non-attacking end of the putrescine/spermidine binding cavity. The current docking studies showed that the aminopropyl chain of compound 9 bind to these distinct pockets, when docked to PfSpdS or $H s S p m S$, which could be explored for inhibitor selectivity between these enzymes.

\section{Conclusions}

This study brings together an array of chemical, biological, biophysical and molecular modeling techniques capturing and explaining the most important characteristics of ligand binding to PfSpdS. Although the computational studies correctly predicted the lowest binding energy pose of PfSpdS inhibitors no clear correlations were observed between the docking scores and inhibitory values of potential and known inhibitors of PfSpdS. The discrepancies could be explained by challenges when docking positively charged flexible polyamines and when working with multi-substrate enzymes. It was essential to incorporate previously reported medicinal chemistry results in this study to guide decision making in the lead discovery process [21,22,54-56]. As a result an in silico predicted binding pose of compound 8 in the presence of the end product, MTA, was confirmed by crystallography studies. Noteworthy is that compound 8 had an unexpected 100 -fold lower $\mathrm{IC}_{50}$ value than compound 9 despite its similarity, which resulted in the reevaluation of the initial inhibition hypothesis. Previously, Shirahata et al. showed compound 9 to be a potent inhibitor of mammalian spermine synthase but not of mammalian SpdS [22,54]. In this study however, compound 9 was identified as a PfSpdS inhibitor with an
$\mathrm{IC}_{50}$ comparable to the most potent known inhibitors of PfSpdS. Because compound 9 did not inhibit mammalian SpdS it makes it the only known inhibitor specific for PfSpdS. Its specificity is attributed to the ability of PfSpdS to alternatively accommodate spermidine to $\mathrm{pu}-$ trescine as substrate for spermine synthesis. The potency of compound 9 was a surprising result given that the rate of spermine synthesis by PfSpdS is only $15 \%$ of the rate of spermidine synthesis [18]. These results provide new insights and opportunities, especially the distinctive binding of the aminopropyl chain of compound 9 to the non-attacking end of the putrescine/spermidine binding cavity, to be explored for the design of selective inhibitors of PfSpdS.

\section{Accession code}

Coordinate and structure factor files for the PfSpdS complex [PDB: 3RIE] were deposited at the Protein Data Bank.

\section{Additional files}

Additional file 1: Additional compounds identified from virtual screening that were docked and tested in vitro against PfSpdS.

Additional file 2: Phase space sampling: A 5 ns molecular dynamics (MD) simulation used to capture the flexibility of the active site.

Additional file 3: An illustration of the conformational change GIn229 undergoes upon binding of 4MCHA and AdoDATO as well as putrescine.

Additional file 4: Average $B$ factor values for the $C_{a}$ backbone and gate-keeping loop of three different PfSpdS crystal structures.

Additional file 5: Inhibition kinetics of PfSpdS treated with compound 9.

Additional file 6: Superimposition of the human spermine synthase (grey ribbon) and PfSpdS (blue ribbon).

Additional file 7: Two different binding poses spermine assumes when co-crystallized with MTA within PfSpdS.

\section{Abbreviations}

AdoDATO: S-adenosyl-1,8-diamino-3-thio-octane; AdoMetDC:

S-adenosylmethionine decarboxylase; APA: 3-aminooxy-1-aminopropane; APE: 5-amino-1-pentene; dcAdoMet: decarboxylated S-adenosylmethionine;

DPM: Dynamic pharmacophore model; EV: Exclusion volume; HBA: Hydrogen bond acceptor; HBD: Hydrogen bond donor; HYD: Hydrophobic;

4MCHA: trans-4-methylcyclohexylamine; MIF: Molecular interaction field; MM-GB/SA: Molecular mechanics generalized born/surface accessible; MTA: 5'-methylthioadenosine; ns: nanosecond; ODC: Ornithine decarboxylase; PBC: Periodic boundary conditions; PhF: Pharmacophore feature; PI: Positive ionizable; PME: Particle mesh Ewald summation; ps: picosecond; SD: Steepest decent; SpdS: Spermidine synthase; Spms: Spermine synthase.

Competing interest

The authors declare that they have no competing interests.

\section{Authors' contributions}

PBB, MW, JS, IBM, FJ, L-MB, and AIL participated in research design; PBB, MW, JS, SBR, MB and IBM conducted experiments; PBB, MW, JS, SBR and MB performed data analysis; PBB, MW, JS, FJ, L-MB and AIL wrote or contributed to the writing of the manuscript; PBB, JS, MW, FJ, L-MB and AIL contributed new reagents. All authors read and approved the final manuscript. 


\section{Acknowledgements}

We thank James Briggs for introducing us to dynamic pharmacophore modelling and the African Centre for Gene Technologies (ACGT) for funding the research visit of PBB. We thank Salam Al-Karadaghi and Lo Persson from Lund University for assistance with crystal growth, beam time and structure solving; Bridgette Cummings and Dina le Roux for assistance with bio-assays. We thank James Snyder from Emory University for the use of the Schrödinger software. PBB and MW were recipients of PhD bursaries from the University of Pretoria (UP) and the South African National Research Foundation (NRF). PBB was supported by a Postdoctoral Fellowship from UP and SBR by an MSc bursary from the South African Malaria Initiative (SAMI). This work was supported by grants from SAMI (LMB) and the NRF (Grants FA2007050300003 and IFR1203060402 to AIL). This work was also supported by a collaborative research exchange grant between the South African National Research Foundation (NRF) and the Swedish International Development Cooperation Agency (SIDA, Swedish Research Links Programme). Any opinion, findings and conclusions or recommendations expressed in this paper are those of the author(s) and therefore the NRF does not accept any liability in regard thereto.

\section{Author details}

'Department of Biochemistry, UP Centre for Sustainable Malaria Control, Faculty of Natural and Agricultural Sciences, University of Pretoria, Private Bag X20, Hatfield 0028, South Africa. ${ }^{2}$ Centre for Molecular Protein Science/ Department of Experimental Medical Science, Lund University, S-221 00/84 Lund, Sweden. ${ }^{3}$ Department of Biochemistry, Bernard-Nocht Institute for Tropical Medicine, Bernard Nocht Strasse 74, Hamburg D-20359, Germany. ${ }^{4}$ Swiss Federal Institute of Intellectual Property, Stauffacherstr. 65/59 g, $\mathrm{CH}-3003$ Bern, Switzerland.

Received: 29 October 2014 Accepted: 18 January 2015

Published online: 05 February 2015

\section{References}

1. Dondorp AM, Nosten F, Yi P, Das D, Phyo AP, Tarning J, et al. Artemisinin resistance in Plasmodium falciparum malaria. N Engl J Med. 2009;361:455-67.

2. Ekland $E H$, Fidock DA. In vitro evaluations of antimalarial drugs and their relevance to clinical outcomes. Int J Parasitol. 2008;38:743-7.

3. Heby O, Persson L, Rentala M. Targeting the polyamine biosynthetic enzymes: A promising approach to therapy of African sleeping sickness, Chagas' disease, and leishmaniasis. Amino Acids. 2007:33:359-66

4. Heby $\mathrm{O}$. Role of polyamines in the control of cell proliferation and differentiation. Differentiation. 1981;19:1-20.

5. Wallace HM, Fraser AV, Hughes A. A perspective of polyamine metabolism. Biochem J. 2003;376:1-14.

6. Müller IB. das Gupta R, Lüersen KL, Wrenger C, Walter RD: Assessing the polyamine metabolism of Plasmodium falciparum as chemotherapeutic target. Mol Biochem Parasitol. 2008;160:1-7.

7. Birkholtz L, Williams M, Niemand J, Louw Al, Persson L, Heby O. Polyamine homoeostasis as a drug target in pathogenic protozoa: peculiarities and possibilities. Biochem J. 2011:438:229-44.

8. Muller $\mathrm{S}$, Coombs GH, Walter RD. Targeting polyamines of parasitic protozoa in chemotherapy. Trends Parasitol. 2001;17:242-9.

9. Kaiser A, Gottwald A, Wiersch C, Lindenthal B, Maier W, Seitz HM. Effect of drugs inhibiting spermidine biosynthesis and metabolism on the in vitro development of Plasmodium falciparum. Parasitol Res. 2001;87:963-72

10. Clark K, Dhoogra M, Louw Al, Birkholtz L. Transcriptional responses of Plasmodium falciparum to a-difluoromethylornithine-induced polyamine depletion. Biol Chem. 2009;389:111-25.

11. Das Gupta R, Krause-Ihle T, Bergmann B, Müller IB, Khomutov A, Müller $\mathrm{S}$, et al. 3-Aminooxy-1-aminopropane and derivatives have an antiproliferative effect on cultured Plasmodium falciparum by decreasing intracellular polyamine concentrations. Antimicrob Agents Chemother. 2005;49:2857-64.

12. Assaraf YG, Golenser J, Spira DT, Bachrach U. Polyamine levels and the activity of their biosynthetic enzymes in human erythrocytes infected with the malarial parasite, Plasmodium falciparum. Biochem J. 1984;222:815-9.
13. Teng R, Junankar PR, Bubb WA, Rae C, Mercier P, Kirk K. Metabolite profiling of the intra-erythrocytic malaria parasite Plasmodium falciparum by $1 \mathrm{H}$ NMR spectroscopy. NMR Biomed. 2008;22:292-302.

14. Molitor IM, Knöbel S, Dang C, Spielmann T, Alléra A, König GM. Translation initiation factor elF-5A from Plasmodium falciparum. Mol Biochem Parasitol. 2004;137:65-74.

15. Igarashi K, Kashiwagi K. Modulation of cellular function by polyamines. Int J Biochem Cell Biol. 2010;42:39-51.

16. Saini P, Eyler DE, Green R, Dever TE. Hypusine-containing protein elF5A promotes translation elongation. Nature. 2009;459:118-21.

17. Frommholz D, Kusch P, Blavid R, Scheer H, Tu J-M, Marcus K, et al. Completing the hypusine pathway in Plasmodium. FEBS J. 2009;276:5881-91.

18. Haider N, Eschbach M, de Souza DS, Gilberger T, Walter RD, Lüersen K. The spermidine synthase of the malaria parasite Plasmodium falciparum: Molecular and biochemical characterisation of the polyamine synthesis enzyme. Mol Biochem Parasitol. 2005;142:224-36.

19. RCSB - Protein Data Bank [www.rcsb.org]

20. Dufe VT, Qui W, Müller IB, Hui R, Walter RD, Al-Karadaghi S. Crystal structure of Plasmodium falciparum spermidine synthase in complex with the substrate decarboxylated S-adenosylmethionine and the potent inhibitors 4MCHA and AdoDATO. J Mol Biol. 2007;373:167-77.

21. Lakanen JR, Pegg AE, Coward JK. Synthesis and biochemical evaluation of adenosylspermidine, a nucleoside-polyamine adduct inhibitor of spermidine synthase. J Med Chem. 1995;38:2714-27.

22. Shirahata A, Morohohi T, Fukai M, Akatsu S, Samejima K. Putrescine or spermidine binding site of aminopropyltransferases and competitive inhibitors. Biochem Pharmacol. 1991;41:205-12.

23. Jacobsson M, Gäredal M, Schultz J, Karlén A. Identification of Plasmodium falciparum spermidine synthase active site binders through structure-based virtual screening. J Med Chem. 2008;51:2777-86

24. Carlson HA, McCammon JA. Accommodating protein flexibility in computational drug design. Mol Pharmacol. 2000;57:213-8.

25. Damm KL, Carlson HA. Exploring experimental sources of multiple protein conformations in structure-based drug design. J Am Chem Soc. 2007;129:8225-35.

26. Vriend G. WHAT IF: A molecular modeling and drug design program. J Mol Graph. 1990;8:52-6.

27. Laskowski RA, MacArthur MW, Moss DS, Thornton JM. PROCHECK: A program to check the stereochemical quality of protein structures. J Appl Cryst. 1993;26:283-91.

28. UHBD [http://web.archive.org/web/20100701074459/http://adrik.bchs.uh. edu/uhbd.html].

29. Krieger E, Nielsen JE, Spronk CAEM, Vriend G. Fast empirical pKa prediction by Ewald summation. J Mol Graph Model. 2006;25:481-6.

30. Phillips JC, Braun R, Wang W, Gumbart J, Tajkhorshid E, Villa E, et al. Scalable molecular dynamics with NAMD. J Comput Chem. 2005;26:1781-802

31. Brooks BR, Bruccoleri RE, Olafson BD, States DJ, Swaminathan S, Karplus M. CHARMM: A program for macromolecular energy, minimisation, and dynamics calculations. J Comput Chem. 1983;4:187-217.

32. Humphrey W, Dalke A, Schulten K. VMD: Visual Molecular Dynamics. J Mol Graph. 1996;14(27-8):33-8.

33. Darden T, York D, Pederson L. Particle Mesh Ewald-an N. Log $(N)$ method for Ewald sums in large systems. J Chem Phys. 1993;98:10089-92.

34. Berendsen HJC, Postma JPM, W.F. Vangunsteren AD, Haak JR. Molecular-dynamics with coupling to an external bath. J Chem Phys. 1984;81:3684-90.

35. Daura X, van Gunsteren WF, Mark AE. Folding-unfolding thermodynamics of a $\beta$-heptapeptide from equilibrium simulations. Proteins. 1999;34:269-80.

36. Van Der Spoel D, Lindahl E, Hess B, Groenhof G, Mark AE, Berendsen HJC. GROMACS: Fast, flexible, and free. J Comput Chem. 2005;26:1701-18.

37. Goodford PJ. A computational procedure for determining energetically favorable binding sites on biologically important macromolecules. J Med Chem. 1985;28:849-57.

38. Pegg AE, Poulin R, Coward JK. Use of aminopropyltransferase inhibitors and of non-metabolizable analogs to study polyamine regulation and function. Int J Biochem Cell Biol. 1995;27:425-42.

39. Goda H, Watanabe T, Takeda N, Kobayashi M, Wada M, Hosoda H, et al. Mammalian spermidine synthase-identification of cysteine residues and investigation of the putrescine binding site-. Biol Pharm Bull. 2004;27:1327-32 
40. SGC Toronto [Www.sgc.utoronto.ca]

41. Kabsch W. XDS. Acta Crystallogr Sect D Biol Crystallogr. 2010;66:125-32.

42. Brunger AT, Adams PD, Clore GM, DeLano WL, Gros P, Grosse-Kunstleve RW, et al. Crystallography \& NMR System: A new software suite for macromolecular structure determination. Acta Crystallogr Sect D Biol Crystallogr. 1998;54:905-21.

43. Vagin AA, Steiner RS, Lebedev AA, Potterton L, McNicholas S, Long F, et al. REFMAC5 dictionary: Organisation of prior chemical knowledge and guidelines for its use. Acta Crystallogr Sect D Biol Crystallogr. 2004:60:2284-95.

44. Emsley P, Lohkamp B, Scott WG, Cowtan K. Features and development of Coot. Acta Crystallogr D Biol Crystallogr. 2010;66:486-501.

45. Schuettelkopf AW, van Aalten DMF. PRODRG-A tool for high-throughput crystallography of protein-ligand complexes. Acta Crystallogr Sect D Biol Crystallogr. 2004;60:1355-63.

46. Lovell SC, Davis IW, Arendall WB, de Bakker PIW, Word JM, Prisant MG, et al. Structure validation by $C a$ geometry: $\varphi, \psi$ and $C \beta$ deviation. Proteins. 2003:50:437-50.

47. Chen VB, Arendall III WB, Headd JJ, Keedy DA, Immormino RM, Kapral GJ, et al. MolProbity: All-atom structure validation for macromolecular crystallography. Acta Crystallogr Sect D Biol Crystallogr. 2010;66:12-21.

48. ADIT deposition tool [http://deposit.pdb.org/validate]

49. Osguthorpe DJ, Sherman W, Hagler AT. Exploring protein flexibility: incorporating structural ensembles from crystal structures and simulation into virtual screening protocols. J Phys Chem B. 2012;116:6952-9.

50. Korolev S, Ikeguchi Y, Skarina T, Beasley S, Arrowsmith C, Edwards A, et al. The crystal structure of spermidine synthase with a multisubstrate adduct inhibitor. Nat Struct Biol. 2002;9:27-31.

51. Wu H, Min J, Ikeguchi $Y$, Zeng $H$, Dong A, Loppnau $P$, et al. Structure and mechanism of spermidine synthases. Biochemistry. 2007;46:8331-9.

52. Khabarova LA, Koikov LN. Comparison of the basicity of quinuclidine, $\mathrm{N}$-methylpiperidine, and triethylamine in water and ethanol-water mixtures. Pharmaceutical Chemistry Journal. 1995:29:786-7.

53. Krissinel $E$, Henrick K. Inference of macromolecular assemblies from crystalline state. J Mol Biol. 2007:372:774-97.

54. Shirahata A, Takahashi N, Beppu T, Hosoda H, Samejima K. Effects of inhibitors of spermidine synthase and spermine synthase on polyamine synthesis in rat tissues. Biochem Pharmacol. 1993;45:1897-903.

55. Shirahata A, Morohoshi T, Samejima K. Trans-4-methylcyclohexylamine, a potent new inhibitor of spermidine synthase. Chem Pharm Bull (Tokyo). 1988;36:3220-2.

56. Tang KC, Mariuza R, Coward JK. Synthesis and evaluation of some stable multisubstrate adducts as specific inhibitors of spermidine synthase. J Med Chem. 1981;24:1277-84.

\section{Submit your next manuscript to BioMed Central and take full advantage of:}

- Convenient online submission

- Thorough peer review

- No space constraints or color figure charges

- Immediate publication on acceptance

- Inclusion in PubMed, CAS, Scopus and Google Scholar

- Research which is freely available for redistribution 